\title{
Two-dimensional MHD model of the reconnection diffusion region
}

\author{
N. V. Erkaev ${ }^{1}$, V. S. Semenov ${ }^{2}$, and H. K. Biernat ${ }^{3}$ \\ ${ }^{1}$ Institute of Computational Modelling, Russian Academy of Sciences, Krasnoyarsk, 660036, Russia \\ 2 Institute of Physics, University of St. Petersburg, St. Petergof, 198504, Russia \\ ${ }^{3}$ Space Research Institute, Austrian Academy of Sciences, Schmiedlstrasse 6, Graz, A-8042, Austria
}

Received: 06 August 2001 - Accepted: 16 October 2001

\begin{abstract}
Magnetic reconnection is an important process providing a fast conversion of magnetic energy into thermal and kinetic plasma energy. In this concern, a key problem is that of the resistive diffusion region where the reconnection process is initiated. In this paper, the diffusion region is associated with a nonuniform conductivity localized to a small region. The nonsteady resistive incompressible MHD equations are solved numerically for the case of symmetric reconnection of antiparallel magnetic fields. A Petschek type steady-state solution is obtained as a result of time relaxation of the reconnection layer structure from an arbitrary initial stage. The structure of the diffusion region is studied for various ratios of maximum and minimum values of the plasma resistivity. The effective length of the diffusion region and the reconnection rate are determined as functions of the length scale and the maximum of the resistivity. For sufficiently small length scale of the resistivity, the reconnection rate is shown to be consistent with Petschek's formula. By increasing the resistivity length scale and decreasing the resistivity maximum, the reconnection layer tends to be wider, and correspondingly, the reconnection rate tends to be more consistent with that of the Parker-Sweet regime.
\end{abstract}

\section{Introduction}

Magnetic reconnection is a physical process in plasmas providing a disruption of current layers and a fast conversion of magnetic energy into another kinds of energy, such as bulk motion of the plasma, heating, and acceleration of particles. This process is considered to be very important in solar, space, and laboratory plasmas (Hones, 1984; Priest, 1985). In the model proposed by Petschek (1964), the whole reconnection area consists of large convective and small diffusion regions (Fig. 1). In the convective region, plasma is accelerated at the slow shock fronts which originate from a small

Correspondence to: $\mathrm{H}$. K. Biernat

(helfried.biernat@kfunigraz.ac.at) diffusion region. The intensity of the reconnection process is characterized by the reconnection rate which is determined by the electric field generated in the diffusion region. Therefore, the diffusion region is a central subject concerning the reconnection problem. An alternative model is that of Sweet (1958) and Parker (1957) who consider magnetic reconnection as a pure diffusive process.

Laboratory experiments of magnetic reconnection ( $\mathrm{Ji}$ et al., 1999), as well as numerical simulations carried out by Biskamp (1986) and Scholer (1989) for a constant resistivity are in favor of the Sweet-Parker solution. On the other hand, in cases of nonuniform resistivity localized to a small region, the numerical simulations (Scholer, 1989; Ugai, 1999) show the evidence of Petschek-type reconnection with plasma acceleration at the shock fronts.

For the convective region, an analytical solution of the ideal MHD equations can be obtained as an asymptotic series in the reconnection rate which is considered to be a small parameter. For the diffusion region, it seems to be impossible to find an analytical solution, and hence a solution has to be obtained numerically (Erkaev et al., 2000). The reconnection rate can be determined by matching the solutions corresponding to the diffusion and convective regions.

The aim of our paper is to study a numerical MHD model of the diffusion region for a nonuniform conductivity, and to obtain the reconnection electric field and the reconnection rate as functions of the amplitude and length scale of the resistivity.

The paper is organized as follows: the basic MHD equations, the shock jump relations, and the outer Petschek solution are described in Sect. 2. In Sect. 3, we present the diffusion region scaling and the boundary layer equations. The initial and the boundary conditions, as well as the numerical scheme for the boundary layer equations, are described in Sect. 4. The results of numerical simulations for the diffusion region are presented in Sect. 5. In Sect. 6, we derive the reconnection rate from the matching procedure. Finally, Sect. 7 is devoted to the summary and discussion. Mathematical details are described in the Appendix. 


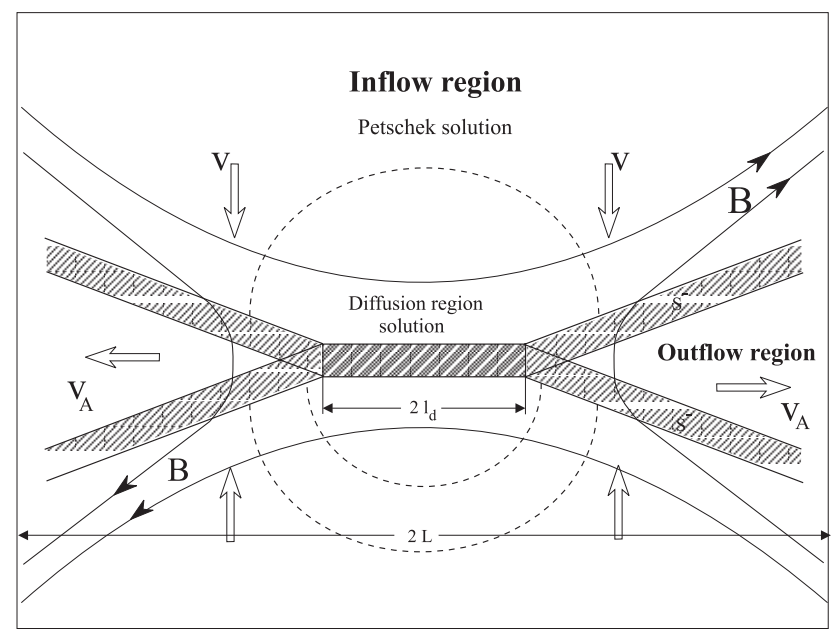

Fig. 1. The Petschek reconnection model: the outer convective and the inner diffusion regions.

\section{MHD equations. Petschek solution}

In our model, the plasma is governed by the resistive steadystate MHD system of equations (Landau and Lifshitz, 1984)

$(\rho \mathbf{v} \cdot \nabla) \mathbf{v}=-\nabla P+\frac{1}{4 \pi}(\mathbf{B} \cdot \nabla) \mathbf{B}$,

$\mathbf{E}+\frac{1}{c}(\mathbf{v} \times \mathbf{B})=\frac{c}{4 \pi \sigma(x, y)} \operatorname{curl} \mathbf{B}$,

$\operatorname{div} \mathbf{B}=0, \operatorname{div} \mathbf{v}=0$,

where incompressibility is assumed; quantity $P$ is the total pressure.

Outside of the diffusion region, dissipation is not important, and we can use the ideal system of MHD equations.

In an incompressible plasma the following relations have to be satisfied at the shock front

$\left\{B_{n}\right\}=0$,

$\left\{v_{n}\right\}=0$,

$\{P\}=0$,

$\left\{\frac{1}{4 \pi} B_{n} \mathbf{B}_{\mathbf{t}}-\rho v_{n} \mathbf{v}_{\mathbf{t}}\right\}=0$,

$\left\{B_{n} \mathbf{v}_{\mathbf{t}}-v_{n} \mathbf{B}_{\mathbf{t}}\right\}=0$,

where the subscripts $n$ and $t$ denote components normal and tangential to the shock front.

Within two dimensions, in Cartesian coordinates $x, y$ ( $x$ is directed along the current sheet), the Petschek solution, which is valid in the convection region, can be presented in a simple analytical form (Petschek, 1964; Vasyliunas, 1975).

Inflow region:

$v_{x}=0, v_{y}=-\varepsilon V_{A}$,

$$
\begin{gathered}
B_{x}=B_{0}-\frac{4 \varepsilon B_{0}}{\pi} \ln \frac{L}{\sqrt{x^{2}+y^{2}}}, \\
B_{y}=\frac{4 \varepsilon B_{0}}{\pi} \arctan \frac{x}{y} .
\end{gathered}
$$

Outflow region:

$v_{x}=V_{A}, v_{y}=0, B_{x}=0, B_{y}=\varepsilon B_{0}$.

The shock front in the first quadrant is described by a linear function

$y=\varepsilon x$.

Just upstream of the shock, the normal magnetic field component is

$B_{y}=2 \varepsilon B_{0}$.

The reconnection rate is defined as

$\varepsilon=E_{0} / E_{A}<<1$,

where $\varepsilon$ is supposed to be a small parameter in the problem. Here $E_{0}$ is the electric field which is constant in the 2D model, and $E_{A}=\frac{1}{c} V_{A} B_{0}$ is the Alfvén electric field, based on $V_{A}=B_{0} /\left(4 \pi \rho_{0}\right)^{1 / 2}$ and $B_{0}$.

Expressions (9)-(13) are asymptotic solutions with respect to $\varepsilon$ (zero and first order terms in the inflow region and only zero order terms in the outflow region) of the ideal MHD system of Eqs. (1)-(3) and the Rankine-Hugoniot shock relations (4)-(8).

Petschek did not obtain a solution for the diffusion region; instead, he suggested an upper estimation for reconnection rate as $\varepsilon \sim 1 / \ln R e_{m}$ which is based on some simple physical ideas. Generally speaking, this implies that the Petschek model gives any reconnection rate from the Sweet-Parker value $1 / \sqrt{R e_{m}}$ up to $1 / \ln R e_{m}$, and for a long time, it was unclear whether Petschek reconnection could be faster than Sweet-Parker reconnection. This problem can be solved by matching a solution for the diffusion region and the Petschek solution ((9)-(13).

\section{Diffusion region scaling}

To obtain the boundary layer MHD equations suitable for the diffusion region, we normalize the MHD parameters to the quantities taken at the upper boundary of the diffusion region

$$
\begin{aligned}
& \tilde{R} e_{m}=4 \pi \sigma^{*} V_{A d} l_{c} / c^{2}, \quad \tilde{x}=x / l_{c}, \\
& \tilde{y}=y / l_{c} \sqrt{\tilde{R} e_{m}}, \quad \tilde{B}_{x}=B_{x} / B_{d}, \\
& \tilde{B}_{y}=B_{y} / B_{d} \sqrt{\tilde{R} e_{m}}, \quad \tilde{V}_{x}=V_{x} / V_{A d} \text {, } \\
& \tilde{V}_{y}=V_{y} / V_{A d} \sqrt{\tilde{R} e_{m}}, \\
& \tilde{E}_{d}=E_{z} c /\left(B_{d} V_{A d}\right) \sqrt{\tilde{R} e_{m}} .
\end{aligned}
$$

Here $\sigma^{*}$ is the minimal conductivity and quantity $l_{c}$ is the length scale of the conductivity. Subscript " $d$ " denotes parameters taken at the upper diffusion region boundary for $x=0$. The local reconnection rate defined as $\tilde{\epsilon}=V_{y d} / V_{A d}$ 
is equal to the normalized electric field $\tilde{E}_{d}$. This scaling for the diffusion region is similar to that for the Prandtl viscous layer (Landau and Lifschitz, 1984) and corresponds exactly to the Sweet-Parker case.

Considering $1 / \tilde{R} e_{m}$ as a small parameter, we obtain the boundary layer equations

$$
\begin{aligned}
& \frac{\partial}{\partial t}\left(\tilde{V}_{x}\right)+\tilde{V}_{x} \frac{\partial}{\partial \tilde{x}}\left(\tilde{V}_{x}\right)+\tilde{V}_{y} \frac{\partial}{\partial \tilde{y}}\left(\tilde{V}_{x}\right) \\
& -\tilde{B}_{x} \frac{\partial}{\partial \tilde{x}}\left(\tilde{B}_{x}\right)-\tilde{B}_{y} \frac{\partial}{\partial \tilde{y}}\left(\tilde{B}_{x}\right)=-\frac{\partial}{\partial \tilde{x}}(\tilde{P}), \\
& \frac{\partial}{\partial \tilde{y}}(\tilde{P})=0 \\
& \frac{\partial}{\partial t}\left(\tilde{B}_{x}\right)=\frac{\partial}{\partial \tilde{y}}\left(\tilde{V}_{x} \tilde{B}_{y}-\tilde{V}_{y} \tilde{B}_{x}\right) \\
& +\frac{\partial}{\partial \tilde{y}}\left(\eta(\tilde{x}, \tilde{y}) \frac{\partial}{\partial \tilde{y}}\left(\tilde{B}_{x}\right)\right), \\
& \frac{\partial}{\partial t}\left(\tilde{B}_{y}\right)=-\frac{\partial}{\partial \tilde{x}}\left(\tilde{V}_{x} \tilde{B}_{y}-\tilde{V}_{y} \tilde{B}_{x}\right) \\
& -\frac{\partial}{\partial \tilde{x}}\left(\eta(\tilde{x}, \tilde{y}) \frac{\partial}{\partial \tilde{y}}\left(\tilde{B}_{x}\right)\right),
\end{aligned}
$$

$\operatorname{div} \tilde{\mathbf{B}}=0$,

$\operatorname{div} \tilde{\mathbf{v}}=0$,

where $\tilde{\eta}(\tilde{x}, \tilde{y})$ is the normalized resistivity of the plasma with the maximum value to be 1 . It can be seen from Eq. (17) that the total pressure is constant across the diffusion region. This is a general feature of a boundary layer approximation.

\section{Initial and boundary conditions. Numerical scheme}

Starting with an initial MHD configuration under fixed boundary conditions, the time-dependent MHD solution converges to a steady state. The normalized total pressure is chosen to be 1 . The internal reconnection rate $\tilde{\varepsilon}$ is to be determined from the numerical solution as a result of time relaxation.

The distribution of the resistivity is traditional (Scholer, 1989; Ugai, 1999)

$\eta(\tilde{x}, \tilde{y})=d e^{\left(-s_{x} \tilde{x}^{2}-s_{y} \tilde{y}^{2}\right)}+f$,

with $d+f=1$.

As the initial configuration, we choose a current sheet with a linear profile of the magnetic field $\tilde{B}_{x}=y, \tilde{B}_{y}=0$. The velocity components are assumed to be equal to zero at the initial moment, $\tilde{V}_{x}=0, \tilde{V}_{y}=0$.

At the upper (inflow) boundary, the tangential magnetic field component is assumed to be constant, $\tilde{B}_{x}=1$, and the tangential velocity component vanishes, $\tilde{V}_{x}=0$. At the left boundary we have the symmetry conditions $\partial\left(\tilde{B}_{x}\right) / \partial x=$ $0, \quad \tilde{B}_{y}=0, \quad \tilde{V}_{x}=0$. At the right boundary we keep free conditions suitable for a uniform flow in the outflow region $\partial\left(\tilde{B}_{y}\right) / \partial x=0, \quad \partial\left(\tilde{V}_{y}\right) / \partial x=0$. At the lower boundary $(y=0)$ there is the symmetry condition for the tangential magnetic field component, $\tilde{B}_{x}=0$, and the non-flow condition for the normal velocity component, $\tilde{V}_{y}=0$. At this boundary, the normal component of the magnetic field $\tilde{B}_{y}$ is obtained from the induction Eq. (19) on the line $y=0$,

$\frac{\partial}{\partial t}\left(\tilde{B}_{y}\right)+\frac{\partial}{\partial x}\left(\tilde{V}_{x} \tilde{B}_{y}\right)=\frac{\partial}{\partial x}\left(\eta J_{z}\right)$

where $J_{z}=\tilde{R} e_{m}^{-1} \partial \tilde{B}_{y} / \partial x-\partial \tilde{B}_{x} / \partial \tilde{y}$. The term $\sim \tilde{R} e_{m}^{-1}$ is included to stabilize the numerical scheme for the unsteady system of the boundary layer MHD equations.

To solve the MHD system numerically, we use a two step conservative finite difference numerical scheme with a rectangular grid $145 \times 100$ in the first quadrant. From each time level $(n)$, we calculate the parameters on the next time level $(n+1)$ in two steps. In the first step $(n+1 / 2)$, diffusion is switched off, and we calculate the parameters for the intermediate time level $(n+1 / 2)$ using the equations in characteristic form. This is similar to the approach used in the Godunov method (Godunov and Ryabenkii, 1987). In the second step, we calculate the parameters for the next time level $(n+1)$ using the equations in conservative form and taking into account the diffusion terms approximated in implicit form. The details of the numerical algorithm are the following. The $\tilde{B}_{x}$ component is found from the $x$-component of the induction equation

$$
\begin{gathered}
\left(\tilde{B}_{x i, k}^{n+1}-\tilde{B}_{x i, k}^{n}\right) / \tau+\left(G_{i, k+1 / 2}^{n+1 / 2}-G_{i, k-1 / 2}^{n+1 / 2}\right) / h x= \\
\left\{\partial / \partial \tilde{y}\left[\eta\left(B_{x} / \partial \tilde{y}-\tilde{R} e_{m}^{-1} \partial \tilde{B}_{y} / \partial \tilde{x}\right)\right]\right\}_{i, k}^{n+1},
\end{gathered}
$$

where quantities

$$
G_{i, k+1 / 2}^{n+1 / 2}=\left(\tilde{B}_{x} \tilde{V}_{y}-\tilde{V}_{x} \tilde{B}_{y}\right)_{i, k+1 / 2}^{n+1 / 2}
$$

are determined by the method of characteristics at the time level $n+1 / 2$.

The normal magnetic field component $\tilde{B}_{y}$ is determined from equation $\operatorname{div} \mathbf{B}=0$ approximated on a rectangular grid. The velocity component $\tilde{V}_{x}$ is found from the $x$-component of the momentum Eq. (16),

$$
\begin{aligned}
& \left(\tilde{V}_{x}^{n+1}-\tilde{V}_{x}^{n}\right)_{i, k} / \tau+\left(Q_{y i, k+1 / 2}-Q_{y i, k-1 / 2}\right)^{n+1 / 2} / h y \\
& \quad+\left(Q_{x i+1 / 2, k}-Q_{y i-1 / 2, k}\right)^{n+1 / 2} / h x=0,
\end{aligned}
$$

where

$$
\begin{gathered}
Q_{y i, k+1 / 2}^{n+1 / 2}=\left(\tilde{V}_{x} \tilde{V}_{y}-\tilde{B}_{x} \tilde{B}_{y}\right)_{i, k+1 / 2}^{n+1 / 2}, \\
Q_{x i+1 / 2, k}^{n+1 / 2}=\left(\tilde{V}_{x}^{2}-\tilde{B}_{x}^{2}\right)_{i+1 / 2, k}^{n+1 / 2} .
\end{gathered}
$$

Here, the parameters ()$_{i, k+1 / 2}^{n+1 / 2}$ are determined by the method of characteristics on the level $n+1 / 2$ simultaneously with the calculation of $\tilde{B}_{x}$. The velocity component $V_{y}$ is determined from equation $\operatorname{div} \mathbf{V}=0$. 


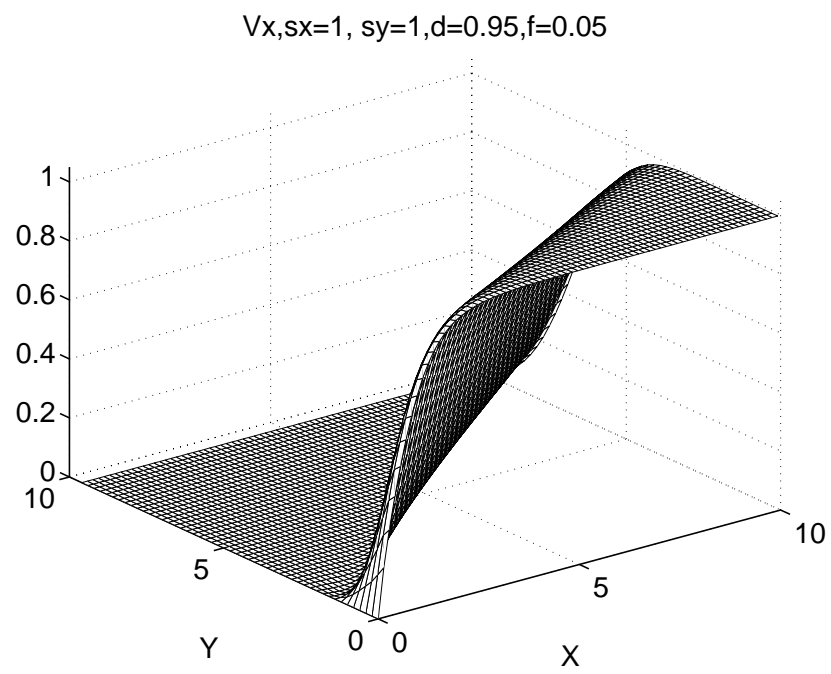

Fig. 2. Tangential velocity component.

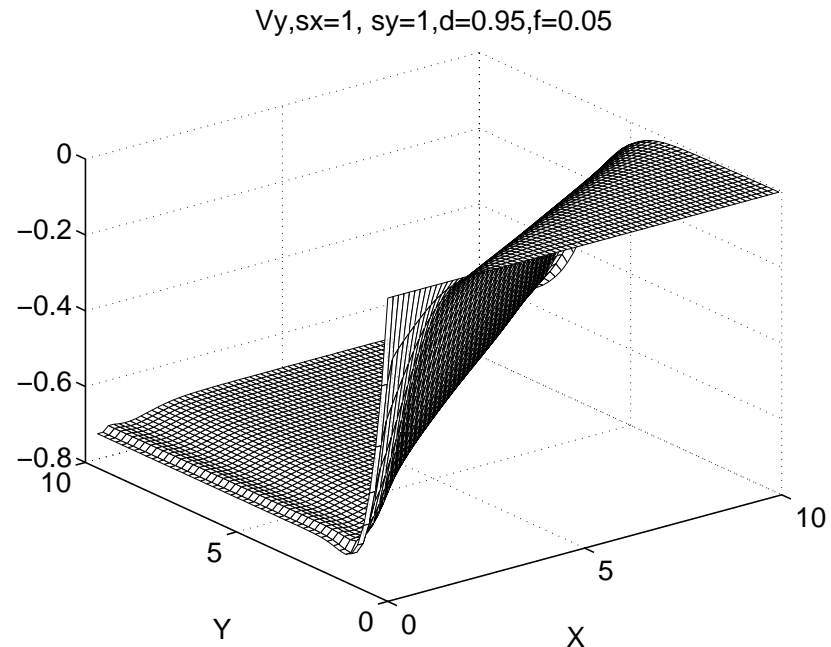

Fig. 3. Normal velocity component.

\section{Results of the numerical simulation}

To estimate the convergence of the time-dependent solution to a steady state for each $n$-th time step, we use the following criteria: $\max \left(\left|V_{x}^{n}-V_{x}^{n-1}\right|\right) /\left(\Delta t\left|V_{x \max }^{n}\right|\right)<10^{-6}$.

For the case of localized resistivity, the system reaches the Petschek steady state with clear asymptotic behaviour (see Figs. 2-7): $V_{x} \rightarrow 1$ in the outflow region; $V_{y} \rightarrow \tilde{\varepsilon}$ at the inflow boundary; $B_{x}$ decreases from 1 to 0 at the shock transition; $B_{y} \rightarrow \tilde{\varepsilon}$ in the outflow region; and $B_{y} \rightarrow 2 \tilde{\varepsilon}$ from the inflow side of the shock (compare with the Petschek solution $(9-12))$.

In the 2D steady state, the total (convective plus dissipative) electric field must be constant. The convective and total electric fields obtained in our calculations are shown in Figs. 6 and 7. One can see that the total electric field is nearly constant except for small perturbations localized to the out-

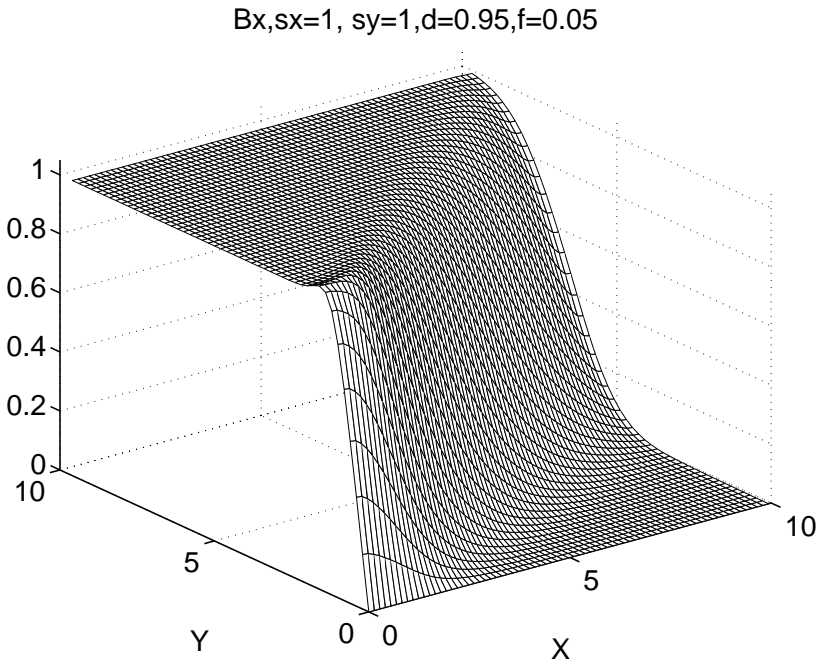

Fig. 4. Tangential magnetic field component.

$B y, s x=1, s y=1, d=0.95, f=0.05$

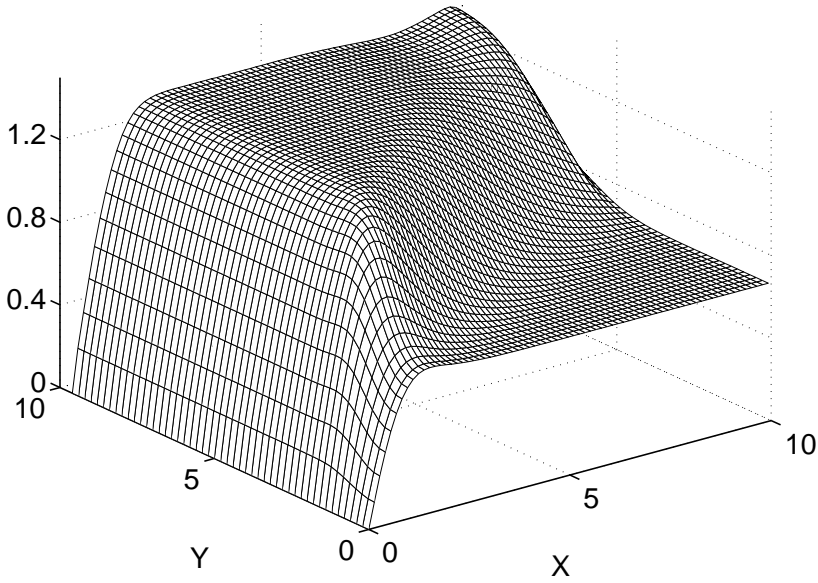

Fig. 5. Normal magnetic field component.

flow boundary due to some influence from the right-hand side boundary conditions.

The magnetic field lines and plasma flow steam lines are shown in Fig. 8. Here, the inflow and outflow regions are clearly separated by the slow shock wave originating from the central part of the diffusion region.

There is a well pronounced slow shock, as can be seen in the behaviour of all MHD parameters. The diffusion region reconnection rate is equal to the normalized diffusion region electric field $\tilde{E}_{d}$ which is obtained to be in the range $0.4<$ $\tilde{E}_{d}<0.7$, as shown in Fig. 9 (top).

On the other hand, for the case of homogeneous resistivity, the system reaches the Sweet-Parker state without any signatures of slow shocks, even if the Petschek solution is used as initial configuration (see also Scholer, 1989; Ugai, 1999; Uzdensky and Kulsrud, 2000). This seems to imply that Petschek-type reconnection is possible only if the resis- 


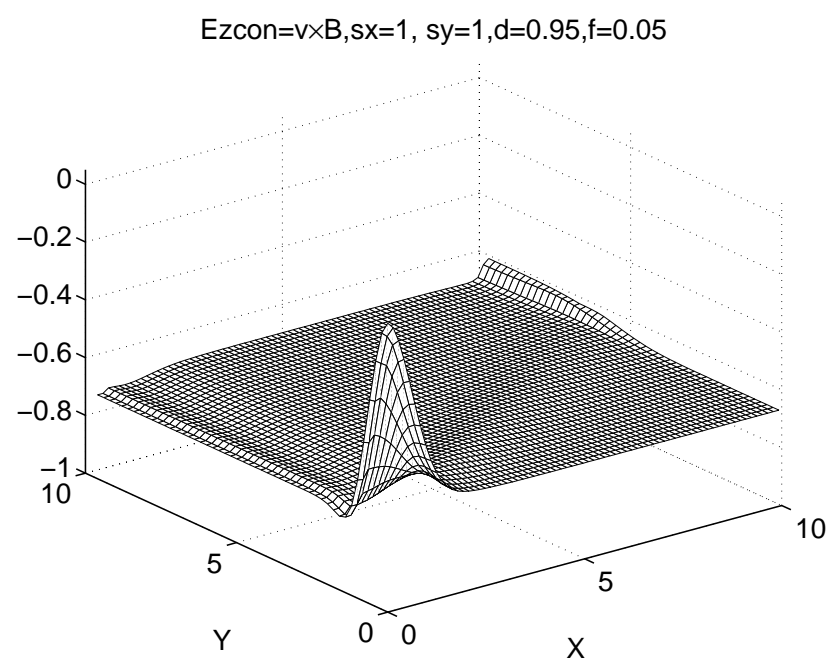

Fig. 6. Convective electric field $-(\mathbf{v} \times \mathbf{B})_{z}$.

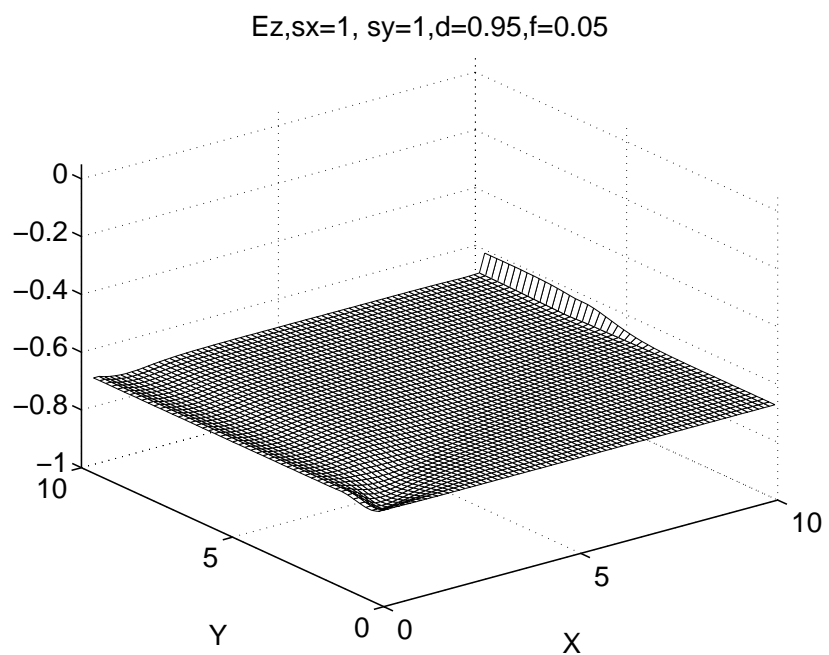

Fig. 7. Total electric field in the diffusion region.

tivity of the plasma is localized to a small region, whereas for constant resistivity, the Sweet-Parker regime is realized (Erkaev et al., 2000).

Figure 9 shows the normalized diffusion region electric field (top) and length scale (bottom) as functions of the parameter $f$. This parameter is introduced as the ratio of the background resistivity to the maximum of the resistivity, and it varies in the range from 0 to 1 . The value 1 corresponds to the case of uniform resistivity.

The length of the diffusion region $l_{d}$, as defined above, is the size of the region where the convective electric field $\mathbf{E}=-\mathbf{v} \times \mathbf{B} / c$ (which is zero at the origin) reaches some level close to the asymptotic value (say 0.9). For the case of localized resistivity, quantity $l_{d}$ practically coincides with the scale of the inhomogeneity of the conductivity when the maximum of the resistivity is much larger than the background resistivity.

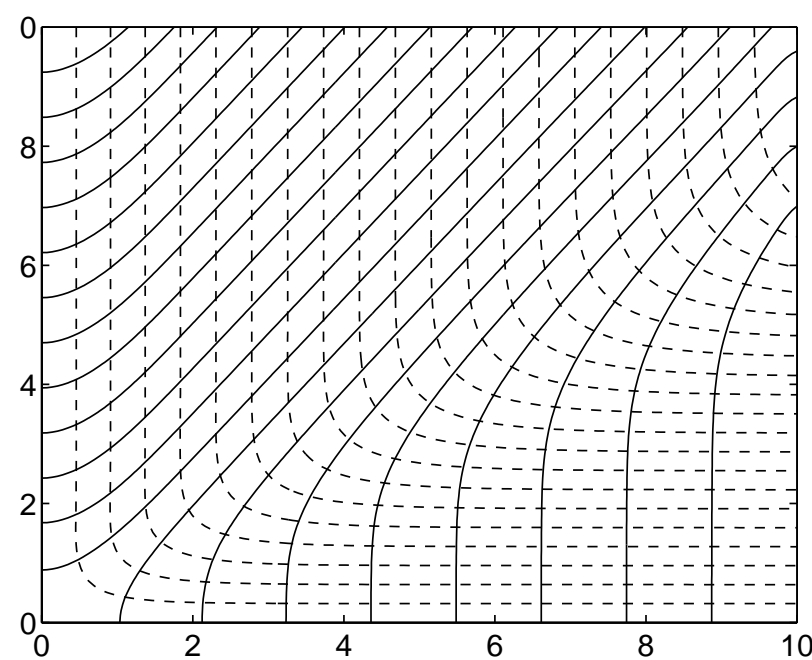

Fig. 8. Magnetic field lines and plasma flow stream lines.
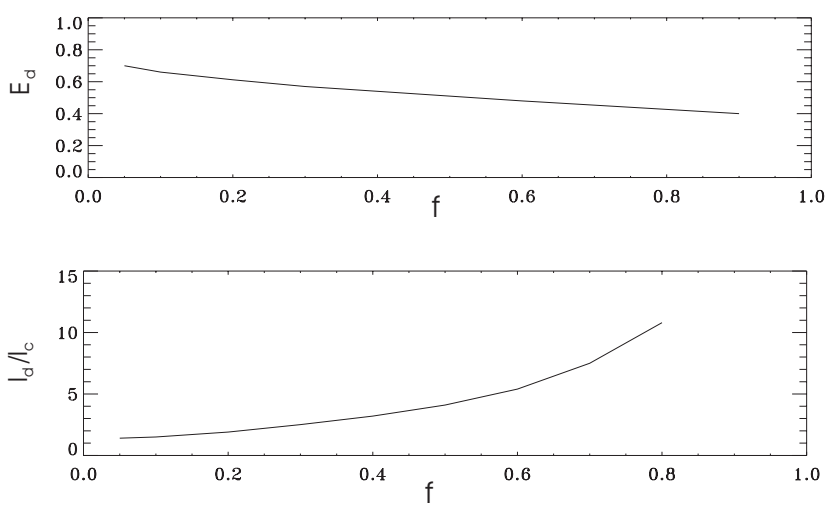

Fig. 9. Electric field and length scale of the diffusion region as functions of the amplitude of conductivity variation.

\section{Reconnection rate}

To find the reconnection rate, we have to determine the magnetic field at the boundary of the diffusion region $B_{d}$ (for details, see Appendix)

$B_{d}=B_{0}\left(1-\frac{4 \varepsilon}{\pi} \ln \frac{L}{l_{d}}\right)$.

The electric field must be constant in the whole inflow region, hence

$v_{y d} B_{d}=v_{0} B_{0}$,

$\epsilon^{\prime} B_{d}^{2}=\varepsilon B_{0}^{2}$,

where the reconnection rates are defined as

$\epsilon^{\prime}=v_{d} / V_{A d}, \quad \varepsilon=v_{0} / V_{A 0}$.

Using the relation $\epsilon^{\prime}=\tilde{\epsilon}\left(\tilde{R} e_{m}\right)^{-1 / 2}$ we obtain

$\tilde{\epsilon} B_{d}{ }^{3 / 2}=\varepsilon B_{0}^{3 / 2} \sqrt{4 \pi \sigma l_{c} B_{0}} / c$. 

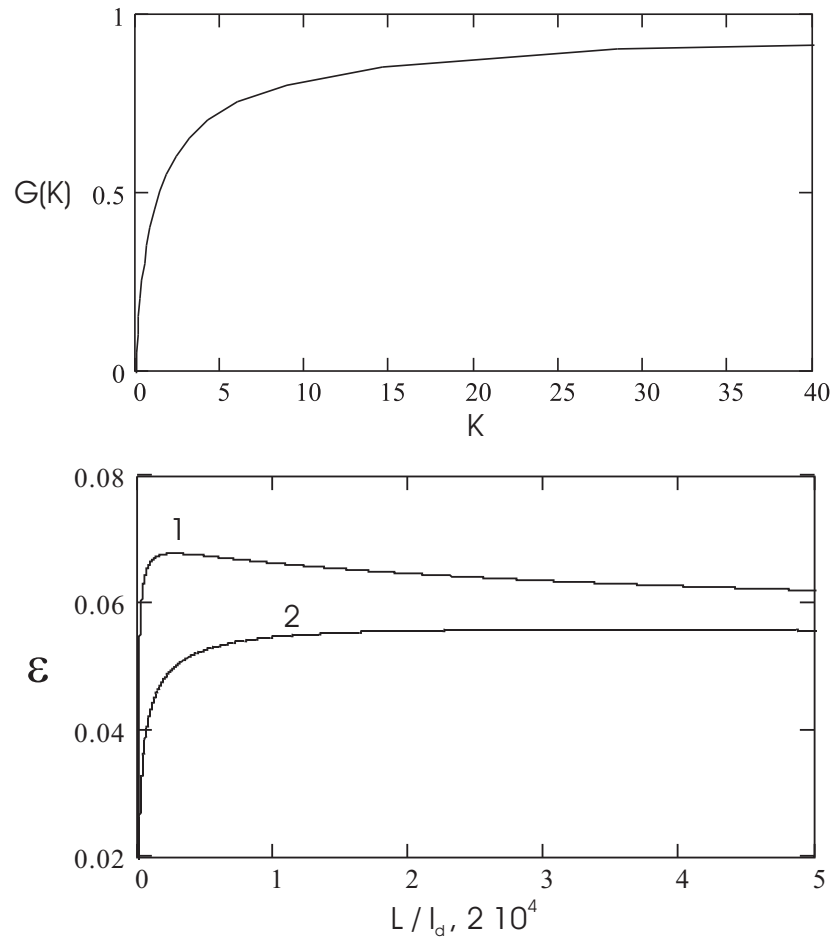

Fig. 10. Function $G(K)$ and the reconnection rate as a function of the ratio $L / l_{d}$. Curves 1,2 correspond to Reynolds numbers $10^{4}$ and $10^{5}$, respectively.

Substituting $B_{d}$ from Eq. (28), we determine finally the following equation for the reconnection rate $\varepsilon$

$\tilde{\epsilon}\left(1-\frac{4 \varepsilon}{\pi} \ln \frac{L}{l_{d}}\right)^{3 / 2}=\varepsilon \sqrt{R e_{m}}\left(\frac{l_{c}}{L}\right)^{1 / 2}$,

where $R e_{m}$ is defined as the magnetic Reynolds number for the global scale and the minimal conductivity, $R e_{m}=4 \pi \sigma^{*} V_{A} L / c^{2}$. Here, the internal normalized reconnection rate $\tilde{\epsilon}$ is obtained from the numerical solution of the diffusion region problem (see Fig. 9), $0.7>\tilde{\epsilon}>0.4$

An important parameter for the reconnection rate is

$K=\frac{4 \ln \left(L / l_{d}\right)}{\pi \sqrt{R e_{m}}}\left(\frac{L}{l_{c}}\right)^{1 / 2}$.

The reconnection rate can be expressed through the function $G(K)$,

$\varepsilon=\frac{\pi}{4} \frac{1}{\ln \left(L / l_{d}\right)} G(K)$,

which is determined implicitly by the nonlinear algebraic equation,

$\tilde{\varepsilon} K[1-G(K)]^{3 / 2}=G(K)$.

The function $G(K)$ is shown in Fig. 10 (top).

For a small value of $K\left(R e_{m}>>1, l_{d} \sim L\right)$, the reconnection rate is similar to that of the Sweet-Parker model.
The reconnection rate, considered as a function of $l_{d} / L$, has a maximum, as shown in Fig. 10 (bottom). For a large parameter $K$, the maximum value $\varepsilon_{\max }$ is proportional to

$1 / \ln \left(R e_{m}\right) \varepsilon_{\max } \sim \frac{\pi}{5} \frac{1}{\ln \left(R e_{m}\right)}$.

This is similar to the Petschek formula.

For small $\varepsilon$ there is a simple analytical expression,

$\varepsilon=\frac{\tilde{\varepsilon}}{\sqrt{R e_{d}}+\frac{6}{\pi} \tilde{\varepsilon} \ln \frac{L}{l_{d}}}$.

Here, $\tilde{\varepsilon}$ is an internal reconnection rate, determined from the numerical solution, which is $\tilde{\varepsilon} \sim 0.7$ for the case of strongly localized resistivity.

It is interesting to note that for the derivation of the final result (32) and (33), the only value which has actually been used, is the internal reconnection rate $\tilde{\varepsilon}$ obtained from the numerical solution. The actual distribution of the $B_{y}$ component along the upper boundary of the diffusion region does not contribute at all to the zero order approximation considered above. Note that in the matching procedure we take into account terms of order $\varepsilon \ln \left(L / l_{d}\right)$, but we neglect terms of order $\varepsilon$.

\section{Conclusions}

The resistive incompressible MHD equations are solved numerically for the case of symmetric reconnection of antiparallel magnetic fields. In the case of nonuniform plasma conductivity localized to a small region, the Petschek type steady-state solution is obtained as a result of the time relaxation of the reconnection layer structure from an arbitrary initial stage. From the mathematical point of view, it is important that the diffusion region solution exists and has the Petschek-like asymptotic behaviour.

The reconnection rate, the effective diffusion region size, and the electric field generated in the diffusion region, are obtained as functions of the amplitude and length scale of the conductivity. The effective length of the diffusion region is obtained as a decreasing function of the resistivity maximum normalized to the background resistivity. For the resistivity maximum approaching the background value, the diffusion region length becomes much longer than the resistivity length scale. On the other hand, for a large resistivity maximum (with respect to the background resistivity), the diffusion region length is of the order of the resistivity length scale.

For a large diffusion region length, the reconnection rate asymptotically tends to that of the Sweet-Parker model. For a small diffusion region length, the reconnection rate is shown to be consistent with Petschek's formula.

The appearance of strongly localized resistivity is often the relevant case in space plasma applications. The efficiency of the Petschek type reconnection is mostly pronounced in cases of very large Reynolds numbers due to the logarithmic 
dependence of the reconnection rate on the Reynolds parameter. In the Petschek model, MHD waves play the dominant role in the conversion of magnetic energy.

Acknowledgements. This work is supported by the INTAS-ESA project 99-01277. It is also supported in part by grants No 01-05-65070 and No 01-05-64954 from the Russian Foundation of Basic Research. Part of this work is supported by the "Fonds zur Förderung der wissenschaftlichen Forschung", project P13804TPH. This work is further supported by grant No 01-05-02003 from the Russian Foundation of Basic Research and by project I.4/2001 from "Österreichischer Akademischer Austauschdienst". We acknowledge support by the Austrian Academy of Sciences, "Verwaltungsstelle für Auslandsbeziehungen".

\section{Appendix. Potential solution in the inflow region}

We consider the inflow region as a semicircle $\left(x^{2}+y^{2} \leq\right.$ $R^{2}, y \geq 0,-R \leq x \leq R$ ). In this region, we have an analytical complex function: $B(z)=B_{y}(x, y)+i B_{x}(x, y)$, where $z=x+i y$, and $B_{x}(x, y), B_{y}(x, y)$ are the magnetic field components which are harmonic functions. For a given function $B_{y}$ at the boundary, we can determine the analytical function inside the semicircle. To find this function, we make a conformal mapping of the semicircle into a unit circle

$w(z)=-\frac{z^{2}+2 i R z+R^{2}}{z^{2}-2 i R z+R^{2}}$.

Inside the circle, the analytical function $B(z)$ is given by (Shabat, 1969)

$B(z)=\frac{1}{2 \pi} \int_{0}^{2 \pi} B_{y}(z(\xi)) \frac{\xi+w}{\xi-w} d t+i C$.

Here, $\xi=\exp (i t)$ and $\mathrm{C}$ are an arbitrary real constant.

The components $B_{x}$ and $B_{y}$ are given by the imaginary and real parts of the integral above, respectively. Thus, for the $B_{x}$ component we find

$B_{x}=\frac{1}{2 \pi} \Im\left(\int_{0}^{2 \pi} B_{y}(z(\xi)) \frac{\xi+w(z)}{\xi-w(z)} d t\right)+C$.

The complex integral can be written as a sum of two integrals

$$
\begin{aligned}
B(z) & =\frac{1}{2 \pi} \int_{-\pi / 2}^{\pi / 2} B_{y}(z(\xi)) \frac{\xi+w(z)}{\xi-w(z)} d t \\
& +\frac{1}{2 \pi} \int_{\pi / 2}^{3 / 2 \pi} B_{y}(z(\xi)) \frac{\xi+w(z)}{\xi-w(z)} d t+i C .
\end{aligned}
$$

Here, the first integral is related to the diffusion region boundary $y=0,-R<x<R$, and the second integral is related to the external boundary $x^{2}+y^{2}=R^{2}, y>0$.

For the external boundary, we take $B_{y}$ from the regularized Petscheck solution,

$$
B_{y}^{*}=\frac{4 \varepsilon B_{0}}{\pi} \arctan \left(\frac{x}{y+l_{d}}\right)
$$

At the diffusion region boundary, the $B_{y}$ component is given by the function

$\left.B_{y}^{d}=\varepsilon B_{0} f\left(x / l_{d}\right)\right)$,

which is different to that given by Eq. (40).

Using (40) and (41), we transform the integral (38) to a sum of two integrals as follows

$$
\begin{aligned}
B_{x} & =\frac{1}{2 \pi} \Im\left(\int_{0}^{2 \pi} B_{y}^{*}(z(\xi)) \frac{\xi+w(z)}{\xi-w(z)} d t\right)+C \\
& +\frac{1}{2 \pi} \Im\left(\int_{-\pi / 2}^{\pi / 2}\left(B_{y}^{d}(z(\xi))-B_{y}^{*}(z(\xi)) \frac{\xi+w(z)}{\xi-w(z)} d t\right) .\right.
\end{aligned}
$$

The first complex integral in (42) can be found by the calculus of residues. We introduce a complex analytical function

$$
F(z)=\frac{4 \varepsilon B_{0}}{\pi}\left(\arctan \left(\frac{x}{y+l_{d}}\right)+i \operatorname{Ln}\left(\sqrt{x^{2}+\left(y+l_{d}\right)^{2}}\right)\right),
$$

which is used for the calculation of the integral as follows

$$
\begin{aligned}
& \frac{1}{2 \pi} \Im\left(\int_{0}^{2 \pi} B_{y}^{*}(z(\xi)) \frac{\xi+w(z)}{\xi-w(z)} d t\right)= \\
& \frac{1}{4 \pi i}\left(\int_{0}^{2 \pi} B_{y}^{*}(z(\xi))\left(\frac{\xi+w(z)}{\xi-w(z)}-\frac{\xi^{*}+w(z)^{*}}{\xi^{*}-w(z)^{*}}\right) d t\right)= \\
& \Re\left(\frac{4 \varepsilon B_{0}}{4 i \pi^{2}}\left(\int_{0}^{2 \pi} F(z(\xi))\left(\frac{\xi+w(z)}{\xi-w(z)}+\frac{\xi+1 / w(z)^{*}}{\xi-1 / w(z)^{*}}\right) d t\right)\right) \\
& =\Re\left(\frac{4 \varepsilon B_{0}}{4 i \pi^{2}}\left(\int_{0}^{2 \pi} F(z(\xi)) \frac{\xi+w(z)}{i \xi(\xi-w(z))} d \xi\right)\right)+ \\
& \Re\left(\frac{4 \varepsilon B_{0}}{4 i \pi^{2}}\left(\int_{0}^{2 \pi} F(z(\xi)) \frac{\xi+1 / w(z)^{*}}{i \xi\left(\xi-1 / w(z)^{*}\right)} d \xi\right)\right) \\
& =\Re\left(\frac{4 \varepsilon B_{0}}{i \pi} F(z)\right)=\frac{4 \varepsilon B_{0}}{\pi} \operatorname{Ln}\left(\sqrt{x^{2}+\left(y+l_{d}\right)^{2}}\right)
\end{aligned}
$$

The constant $\mathrm{C}$ has to be chosen to match the function (42) with the Petschek solution.

Finally, from (42) we obtain

$$
\begin{aligned}
B_{x} & =\frac{4 \varepsilon B_{0}}{\pi} \ln \left(\sqrt{x^{2}+\left(y+l_{d}\right)^{2}} / L\right)+B_{0} \\
& +\frac{1}{2 \pi} \Im\left(\int_{-\pi / 2}^{\pi / 2}\left(B_{y}^{d}(z(\xi))-B_{y}^{*}(z(\xi)) \frac{\xi+w(z)}{\xi-w(z)} d t\right) .\right.
\end{aligned}
$$

For large $R\left(R>>l_{d}\right)$, one can use a Taylor-series expansion of the function $w(z)$

$$
\begin{aligned}
& w(z)=-\frac{1+2 i z / R+O\left((z / R)^{2}\right)}{1-2 i z / R+O\left((z / R)^{2}\right)} \\
& =-\left(1+4 i z / R+O\left((z / R)^{2}\right)\right), \\
& \xi=\exp (i t)=-\left(1+4 i s / R+O\left((s / R)^{2}\right)\right), \\
& \frac{d t}{d s}=\frac{4}{R}+O\left((s / R)^{2} .\right.
\end{aligned}
$$


With the expansion above, the integral in Eq. (44) asymptotically tends to the Poisson integral

$$
\begin{gathered}
\frac{1}{2 \pi} \int_{-\pi / 2}^{\pi / 2}\left(B_{y}^{d}(z(\xi))-B_{y}^{*}(z(\xi))\right) \frac{\xi+w(z)}{\xi-w(z)} d t= \\
\frac{1}{\pi} \int_{-\infty}^{\infty}\left(B_{y}^{d}(s)-B_{y}^{*}(s)\right) \frac{(x-s)}{(x-s)^{2}+y^{2}} d s .
\end{gathered}
$$

Using this asymptotic relation, we finally obtain

$$
\begin{aligned}
& B_{x}= \frac{4 \varepsilon B_{0}}{\pi} \ln \left(\sqrt{x^{2}+\left(y+l_{d}\right)^{2}} / L\right)+B_{0}+ \\
&\left.\frac{1}{\pi} \int_{-\infty}^{\infty}\left(B_{y}^{d}(s)\right)-B_{y}^{*}(s)\right) \frac{(x-s)}{(x-s)^{2}+y^{2}} d s .
\end{aligned}
$$

Thus, the magnetic field at the center of the diffusion region boundary $(x=0, y=0)$ is equal to

$$
\begin{aligned}
B_{0}^{d} & =-\frac{4 \varepsilon B_{0}}{\pi} \operatorname{Ln}\left(L / l_{d}\right)+B_{0}- \\
& \frac{\varepsilon B_{0}}{\pi} \int_{-\infty}^{\infty}\left(f(s)-\frac{4}{\pi} \arctan (s /(s+1))\right) \frac{1}{s} d s .
\end{aligned}
$$

The function

$$
f(s)-\frac{4}{\pi} \arctan (s /(s+1))
$$

must have a zero limit for $s \rightarrow \pm \infty$ because of the matching condition. In the relation (51) we take into account the first two main terms, and neglect the third term.

\section{References}

Biskamp, D.: Magnetic reconnection via current sheets, Phys. Fluids, 29, 1520, 1986.

Erkaev, N. V., Semenov, V. S., and Jamitzky, F.: Reconnection rate for the inhomogeneous resistivity Peschek model, Phys. Rev. Lett., 84, 1455, 2000.

Galeev, A. A., Kuznetsova, M. M., and Zelenyi, L. M.: Magnetopause stability threshhold for patchy reconnection, Space Sci. Rev., 44, 1, 1986.

Godunov S. K. and Ryabenkii V. S.: Difference Schemes: an Introduction to the Underlying Theory, 489, Amsterdam: NorthHolland, 1987.

Hones, E. W., Jr.: Magnetic Reconnection in Space and Laboratory Plasmas, Geophysical Monograph 30, AGU, Washington, 1984.

Ji, H., Yamada, M., Hsu, S., Kulsrud, R., Carter, T., and Zaharia, S. Magnetic reconnection with Sweet-Parker characteristics in twodimensional laboratory plasmas, Phys. Plasmas, 6, 1743, 1999.

Landau L. D. and Lifschitz, E. M.: Lehrbuch der Theoretischen Physik, Klassische Feldtheorie, Akademi-Verlag, Berlin, 1984.

Parker, E. N.: Sweet's mechanism for merging magnetic fields in conducting fluids, J. Geophys. Res, 62, 509, 1957.

Petschek, H. E.: Magnetic field annihilation, NASA Spec. Publ., SP-50, 425, 1964.

Priest, E. R.: The magnetohydrodynamics of current sheets, Rep. Progr. Phys., 48, 9551985.

Pudovkin, M. I. and Semenov, V. S.: Magnetic field reconnection theory and the solar wind-magnetosphere interaction: A review, Space Science Reviews, 41, 1, 1985.

Scholer, M.: Undriven reconnection in an isolated current sheet, J. Geophys. Res., 94, 8805, 1989.

Sweet, P. A.: Electromagnetic Phenomena in Cosmic Physics, edited by B. Lehnert, Cambridge University Press, 123, 1958.

Shabat B. V.: Introduction to complex analysis. Moscow, "Nauka", 1969.

Ugai, M.: Computer studies on the spontaneous fast reconnection model as a nonlinear instability, Phys. Plasmas, 6, 1522, 1999.

Uzdensky, D. A. and Kulsrud, R. M.: A numerical simulation of the reconnection layer in 2D resistive MHD, Phys. Plasmas, 6, 7, 10, 4018-4030, 2000.

Vasyliunas, V. M.: Theoretical models of magnetic field line merging, 1, Rev. Geophys. Space Phys., 13, 303, 1975. 